\title{
State Of The Art: A Study of Human-Robot Interaction in Healthcare
}

\author{
Iroju Olaronke \\ Department of Computer Science, Adeyemi College of Education, Ondo, Nigeria \\ Email: irojuolaronke@gmail.com \\ Ojerinde Oluwaseun and Ikono Rhoda \\ Department of Computer Science, Federal University of Technology, Minna, Nigeria \\ Department of Computer Science and Engineering, Obafemi Awolowo University, Ile-Ife, Nigeria \\ Email: \{o.ojerinde@futminna.edu.ng,rhoda_u@yahoo.com\}
}

\begin{abstract}
In general, the applications of robots have shifted rapidly from industrial uses to social uses. This provides robots with the ability to naturally interact with human beings and socially fit into the human environment. The deployment of social robots in the healthcare system is becoming extensive as a result of the shortage of healthcare professionals, rising costs of healthcare and the exponential growth in the number of vulnerable populations such as the sick, the aged and children with developmental disabilities. Consequently, social robots are used in healthcare for providing health education and entertainment for patients in the hospital and for providing aids for the sick and aged. They are also used for dispensing drugs and providing rehabilitation as well as emotional and aging care. Hence, social robots improve the efficiency and quality of healthcare services. The interaction between social robots and human beings is known as human-robot interaction. Human-robot interaction in healthcare is faced with numerous challenges such as the fear of displacement of caregivers by robots, safety, usefulness, acceptability as well as appropriateness. These challenges ultimately lead to a low rate of acceptance of the robotic technology. Consequently, this paper extensively appraises humanrobot interaction in healthcare, their applications and challenges. Design, ethical and usability issues such as privacy, trust, safety, users' attitude, culture, robot morphology as well as emotions and deception arising from the interaction between humans and robots in healthcare are also reviewed in this paper.
\end{abstract}

Index Terms-Artificial Intelligence, Healthcare, Human-robot interaction, Robots, Social Robots.

\section{INTRODUCTION}

The rising cost of healthcare, the exponential growth of vulnerable population such as the sick and the aged and the shortage of qualified healthcare professionals in recent times has led to logical alternatives of providing healthcare services to patients [1]. One of the major means of providing alternative care is the use of social robots which is fast becoming prevalent as a result of the advancement in robotic technology and Information and Communication Technology (ICT). Social robots are used in healthcare to provide assistive health technology such as aids for the blind, robot wheelchairs and walkers. They are used to rehabilitate the aged and the infirm; they provide remote surgical operations and also dispense oral drugs in pharmaceutical settings [2]. In addition, social robotic systems are used to imitate the cognition of human beings and animals in order to provide companion for the aged. This mitigates boredom, isolation and depression and facilitates the quick recovery of the sick [3]. Social robots possess the ability to stimulate the development of new treatments for different diseases, improve the accessibility to care and also increase independent living. Consequently, social robots improve the quality of healthcare services and patient health outcomes.

One of the major characteristics of a social robot is its ability to naturally interact with human beings and socially fit into the human environment. Furthermore, social robots are autonomous in nature and they have the ability to establish and maintain social relationships with their users [4]. Therefore a social robot according to Weiss and Evers [5] is an embodied intelligent agent which is specifically designed for social interaction with human beings. The interdisciplinary study of the dynamic interaction between human beings and social robots is referred to as human-robot interaction (HRI) [6]. Humanrobot interaction is an emerging field which is multidisciplinary in nature. It is a branch of Computer Science which draws its research links from the field of Artificial Intelligence majorly in Human-Computer Interaction (HCI), Robotic Engineering, Natural Language Processing, and Computer Vision. It is also related to Electrical, Mechanical, Industrial and Design Engineering. Human-robot interaction is as well rooted in Social Sciences majorly in the fields of Human Factors, Psychology, Cognitive Science, Communications, Sociology and Anthropology. It is also associated with Ethology, Ethics, Linguistics and Philosophy in Humanities.

One of the major challenges confronting human-robot interaction in healthcare is the loss of privacy. This is 
because social robots are mobile, they act as social actors and they also have the ability to gather data [7]. Other issues affecting human-robot interaction in healthcare include lack of trust, low user acceptance, emotions and deception. The interaction between humans and social robots within the healthcare system is also faced with the challenge of patients' safety. For instance, during humanrobot interaction, the human is considered as an integral part of a closed-loop feedback system which exchange information and energy with the robot system simultaneously [8]. At this point, too much energy may be transferred by the robot to the human/patient and this might result in severe injury [9]. In addition, the presence of sharp edges in the mechanical design of a social robot increases lacerations which can also cause severe damages to humans [9]. Thus, the social and ethical implications of a social robot must be considered during the design of social robots in healthcare. Hence, this study examines the general overview of robots and the types of robots depending on the type of tasks that they perform. Consequently, industrial robots, service robots and social robots are critically examined in this paper. This study also examines human-robot interaction in healthcare, its applications as well as its challenges. Ethical, design and usability issues associated with the interaction between humans and robots in healthcare are also considered in this study.

This paper is organized into seven sections. Section two deals with the general overview of robots, section three examines human-robot interaction, section four appraises human-robot interaction in healthcare, section five examines the challenges of human-robot interaction in healthcare, section six suggests ways of enhancing effective human-robot interaction in healthcare while section seven concludes the study.

\section{GENERAL OVERVIEW OF RoBOtS}

The term robot originated from the Czech word "robota" which denotes forced labor. The word robot was coined by a Czech novelist named Karel Capek [10]. Capek used the word robot in a 1921 play titled Rossum's Universal Robots (R.U.R.). In R.U.R, robots were manmade beings that were created to work for people. Thus, human beings have used robots to perform different tasks since ancient times. However, there is no standard definition for robots. Nevertheless, the Robot Institute of America [11] defines a robot as a reprogrammable, multifunctional manipulator designed to move material parts, tools or specialized devices through variable programmed motions for the performance of a variety of tasks. Re-programmability in this definition distinguishes robots from other automatic machines [12]. Davison [13] also views a robot as a physically-embodied, artificially intelligent device that has the ability to sense and actuate. Furthermore, Hegel et al. [14] defines a robot as a programmed physical entity that perceives and acts autonomously within a physical environment which has an influence on its behavior. In contrast to Hegel et al. [14] definition, a robot can be fully controlled by a human being, that is, teleoperated. From the definitions above, a system or device is considered a robot if it possesses the following features:

- Sensing: A robot has the ability to sense its environment. A robot should be able to react and adapt to changing conditions in its environment. A robot should also be able to detect objects or features in its environment.

- Movement: A robot must possess the ability to move in its environment. This could be done by rolling on wheels, walking with legs or propelling by thrusters.

- Energy: A robot must have a source of power such as electrical or solar power.

- Intelligence: A robot must be cognitive in nature by possessing the capability to reason, plan, navigate and manipulate in its environment. A robot also possesses the ability to be easily programmable so that it can perform its tasks.

- Shape: A robot must have a shape, frame or form that is required to carry out a specific task.

A robot can therefore explicitly be defined as a reprogrammable, physically embodied, intelligent and mobile system that is energy driven and has the ability to act autonomously or be teleoperated in an environment which it has the capability to sense. A robot consists of seven basic components irrespective of its shape and size. These components work together to perform a specific task. The components of a robot include the following:

- Controller: The controller coordinates the movement of the robot. The region of space a robot can reach is called the working envelope. The controller is also responsible for receiving input from the environment through its sensors.

- Power conversion unit: The power source provides energy to drive the robot's controller. The most common sources of power in robotic systems include electric power, compressed gases, solar power and hydraulics. The power supplied is usually converted from alternating current (ac) to direct current $(\mathrm{dc})$ in the power conversion unit.

- Manipulator: Robots have the capability to manipulate objects by picking up objects, modifying objects as well as destroying objects. The manipulator is the mechanical handling device of the robot which emulates the arm of a human being. The manipulator consists of a set of rigid links connected by joints which are usually referred to as the shoulder, elbow and wrist. The joints are usually rotary or sliding in nature. The arrangement of the joints which determines the possible motion of the robot is referred to as kinematics.

- End Effector: The last link of the manipulator is called the end effector. The effector is defined as a link that is used to grip a tool. The end effector emulates the human hand. 
- Sensors: The sensors allow robots to receive information about a certain measurement of the environment. This is usually done to ensure the safety of the robot. Sensors allow a robot to act on changes in the environment.

- Actuator: The actuator is usually referred to as the muscle of the robot. It converts the power supplied into the robot's movement.

- Control and Task Program: The control program is a set of instructions provided by the manufacturer of the robot to control the robot's manipulator while the task program is a set of instructions usually provided by the user. The task program specifies the motion that the manipulator needs to complete a specific task.

Hence, Qureshi and Syed [15] view a robot as a system that contains sensors, control systems, manipulators, power supplies, and software that work together to perform a specific task. The components of a robot are as illustrated in Fig. 1.

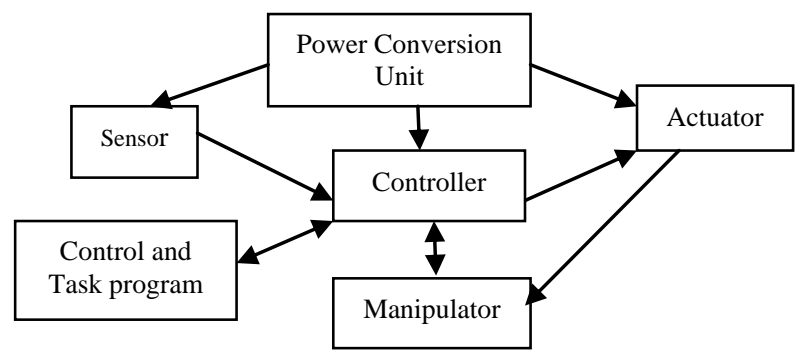

Fig.1. Components of a robot [11]

Robots can be classified into different categories depending on the task that they perform. Based on this, the International Federation of Robots classified robots into two basic classes. These include the industrial robots and the service robot [16].

\section{A. Industrial Robots}

An industrial robot according to the International Federation of Robots is an automatically controlled, reprogrammable, multipurpose manipulator that is programmable in three or more axes which may be either fixed or mobile, and designed for use in industrial automation applications [17]. Industrial robots are usually used in manufacturing industries to perform tasks that are too cumbersome or too dangerous for human beings. They are mostly found in automobile industries where they are used to perform repetitive and predictable tasks. The applications of industrial robots typically include painting, welding, assembling, picking and placing, palletizing, product inspection and testing. Most industrial robots are robot arms or manipulators whose function is to position an end-effector through which it interacts with its environment. However, industrial robots are designed for environments where the presence of human beings is limited [18].

\section{B. Service Robots}

Engelhardt [19] views a service robot as a system that functions as a smart, programmable tool that can sense, think, and act to benefit or enable humans to extend/enhance human productivity. In addition, the International Federation of Robots defines a service robot as a robot which operates semi autonomously or fully autonomously to perform tasks that are useful to the well being of human beings and equipment, excluding manufacturing operations [17]. It is clear from the above definitions that service robots are not used for manufacturing purposes and they do not interact with human beings. Conversely, a third class of robots was established. This special type of robot known as social robot was specifically designed to interact socially with human beings and other robots in order to support a human-like interaction.

\section{Social Robots}

A social robot according to Bartneck and Forlizzi [20] is an autonomous or semi-autonomous robot that interacts with human beings by following the behavioral norms expected by the people with whom the robot is intended to interact. However, Breazeal [21] argues that social robots interact with both human beings and other robots. Fong et al. [22] also corroborated Breazeal [21] by defining social robots as embodied agents that are part of a heterogeneous group of a society of robots or humans. However, for a robot to be considered social it must possess the following characteristics:

- Social Interaction: A social robot engages in social interactions by explicitly communicating with human beings within the social rules attached to its roles. Hence, Fong et al. [22] emphasized that social robots have the ability to establish and maintain social relationships with human beings using natural cues such as gaze and gestures. Breazeal [21] stressed that human beings should be able to understand a social robot, relate with it and also empathize with it. Social robots also have the ability to express and perceive emotions. Hence, social robots exhibit personality, traits and character.

- Anthropomorphism: Anthropomorphism is a phenomenon that describes the tendency of humans to see human-like shapes in an environment [23]. It is a process of attributing human like qualities and personal characteristics to entities in an environment. According to Graaf et al. [4], social robots can be designed to possess life-like qualities in order to enhance their interactions with human beings. Robots that take the shape and possess the qualities of humans are referred as humanoid robots.

- Social learning and imitation: A social robot can easily adapt, perceive, recognize and learn new behaviors or skills by imitation through natural or intuitive means [18]. Behaviors can be verbal or non-verbal. Examples of non-verbal behaviors include gestures and gazing. 
One of the basic requirements that manage the behavior of social robots is the Isaac Asimov three laws of robots [24]. These laws are highlighted below.

- A robot may not injure a human being or through inaction allow a human being to come to harm.

- A robot must obey orders given to it by human beings except where such orders would conflict with the first law.

- A robot must protect its own existence as long as such protection does not conflict with the first or second law.

These three laws prove that the closer a human gets with a robot, the more complicated their relationship becomes and the risk of the human getting injured is heightened. Hence, safe zones must be defined during human-robot interaction. The basic consequence of these laws however is that robots that interact with other robots are not considered social robots. This is however against the definitions of Breazeal [21] and Fong et al. [22] which argued that social robots interact with both humans and other robots. However, Vincent et al. [25] is of the view that a robot will only be considered social if it interacts with humans within the social values, norms and standards of a society. Consequently, Vincent et al. [25] view robots as culturally dependent since social values, norms and standards differ amongst cultures.

\section{HUMAN-ROBOT INTERACTION}

There are diverse definitions for human-robot interaction. For instance, Goodrich and Schultz [26] define human-robot interaction (HRI) as a field of study that is dedicated to understanding, designing, and evaluating robotic systems for use by or with humans. Interaction in this definition according to Goodrich and Schultz [26] requires communication between robots and humans. In addition, Feil-Seifer and Mataric [6] view
HRI as an interdisciplinary study of the dynamic interaction between human beings and robots. Interaction in this definition refers to the process of working together to achieve a common goal. HRI focuses on the study of the functionality and the usability of robots when performing tasks that involve human beings [27]. There are diverse means in which a human and a robot interact. These include the use of visual displays such as graphical user interfaces or augmented reality interfaces, gaze and gestures such as hand and facial movements, speech, natural languages, physical interaction and haptics [26]. HRI is therefore focused on making the interactions between robots and human beings as natural as possible. However, it is important to note that the social interaction between humans and robots is not limited to one human and one robot. The interactions between humans and robots in HRI can also take the form of one human-robot team, one human-multiple robots, human team-one robot, multiple humans-one robot, human team-robot team, human team-multiple robots, multiple humans-robot team [28]. A team in this regard refers to a group of humans or robots working together to achieve a common goal. The robot team could however contain different types of robots or the same type of robot. The form of interactions between humans and robots is as illustrated in Fig. 2.

According to Goodrich and Schultz [26], the interaction between a human and robot are categorized into two. These include remote interaction and proximate interaction.

- Remote interaction: As the name implies, in remote interaction the human and the robot are not located in the same geographical location. The humans and the robots may be separated spatially or temporally.

- Proximate interaction: In proximate interaction, the humans and the robots are in the same location or environment. This implies that the humans and robots are collocated.

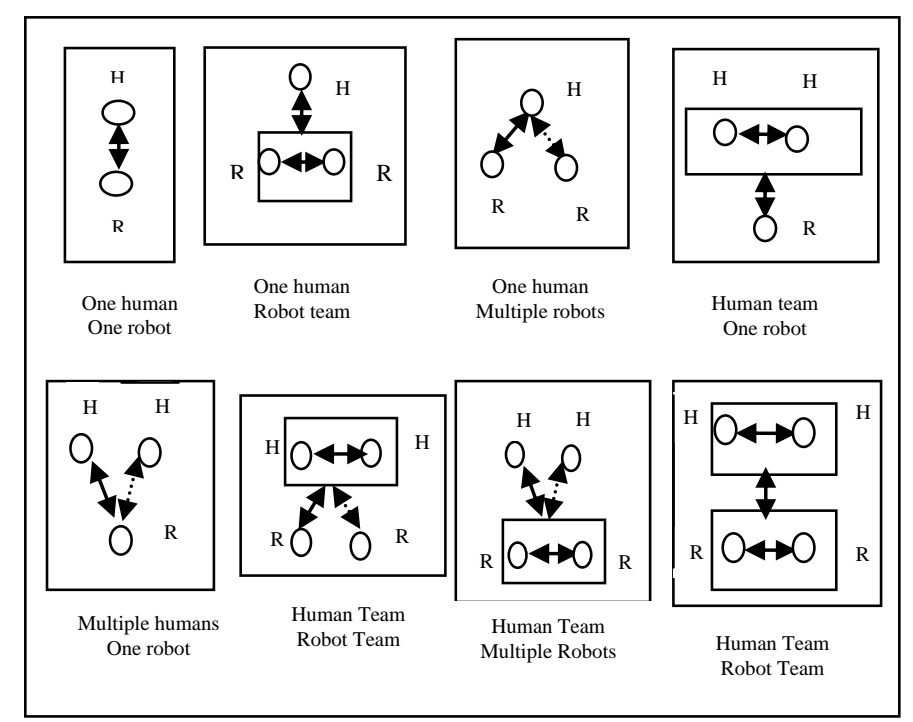

Fig.2. Forms of interactions in HRI [28] 
According to Yanco and Drury [28], there are different types of interaction roles in HRI. These include:

- Supervisor: A supervisor role involves monitoring, controlling and evaluating a task that is required to be performed by a robot. Hence, the supervisor controls the behavior of the robot. The supervisor can manage more than one robot. The human usually performs the role of a supervisor in HRI.

- Operator: The operator is responsible for knowing where a robot is and what the robot is doing at any given time. The operator also has the knowledge of the robot's health and environment. The operator is also tasked with the responsibility of modifying the robot's behavior to a suitable one. The person who is responsible for remotely operating and controlling a robot is known as the Wizard-of-Oz (WoZ).

- Mechanic: The mechanic plays the role of the programmer. The mechanic is responsible for changing the robots' hardware or software.

- Peer/Team: The word peer refers to teammates. Hence, a robot as well as a human can be a member of a team. Both the human and the robot in the team work together to achieve a common objective. Interactions between the robot and the human at this point could be through gestures, gaze and voice.

- Bystander: The role of the bystander is to coexist in the same environment with a robot without necessarily interacting with the robot. However, the bystander is required to have a little knowledge of the robot's behavior so as to understand the consequences of the robot's action.

Hence, both humans and robots must have the knowledge of one another. This process is referred to as Human-Robot Interaction Awareness. Formally, Drury et al. [29] defines HRI awareness as the understanding that the human has of a robot's location, activities, status, and surroundings; the knowledge that the robot has of the human's commands or instructions that are necessary to direct its activities and the knowledge of the conditions and constraints under which the robot must operate. However, the lack of awareness significantly reduces the level of interaction between a human and a robot and thus the performance of the overall task required to be carried by both the human and the robot is greatly reduced [30]. According to Drury et al. [29], there are five types of HRI awareness. These include the following:

- Human-robot Awareness: In human-robot awareness, the human have the knowledge of the robots' locations, surroundings, identities, activities and status.

- Human-human Awareness: In human-human awareness, the humans have the knowledge of the locations, surroundings, identities, activities and status of their fellow human collaborators.
- Robot-human Awareness: In robot-human awareness, the robots have the knowledge of the humans' instructions needed to perform a specific task.

- Robot-robot Awareness: In robot-robot awareness, the robots' have the knowledge of the instructions given to them by other robots.

- Humans' overall mission Awareness: In humans' overall mission awareness, the human beings have the knowledge of the overall goal of the mutual activities carried out by the humans and the robots.

There are five basic taxonomies of interactions between humans and robots [26]. These taxonomies include autonomy, the nature of information exchange, the structure of the team, adaptation, learning, and training of people and the robot as well as the shape of the task.

- Autonomy: Autonomy can simply be described as the ability of a robot to carry out a task independently. Four levels of autonomy have been identified by the Department of Defense, United States of America [17]. These include human operated, human delegated, human supervised and fully autonomous. In human operated autonomy, the robot has no autonomous control of its environment while the human operator makes all the decisions. In human delegated autonomy, the robot performs diverse tasks which are delegated to it, independent of human control. In human supervised autonomy, the robot performs diverse tasks when directed by humans while in fully autonomous autonomy, the robot receives goals from the human and translates them into tasks to be performed without requiring human interaction, although the human can still change the goal in this process.

- Nature of Information Exchange: The nature of information exchange in this context refers to the way or manner in which humans and robots exchange information. Information is usually exchanged between humans and robots using different types of communication media which are characterized by the senses of hearing, touch and seeing [26]. Typical media of communication used for information exchange between humans and robots include gestures, such as hand and facial movements, speech, natural languages and visual displays such as graphical user interfaces or augmented reality interfaces.

- The structure of the team: This can simply be described as the number, the composition and the organization of humans and robots that are working together to perform a particular task

- Adaptation, Learning and Training: This simply means that a robot has the ability to be trained or taught a particular behavior and it also has the ability to learn new behaviors by natural or 
intuitive means. A robot also possesses the ability to adapt to changes in its environment. In HRI, humans who are relatively inexperienced in a particular task can also trained.

- Shape of the Task: The shape of the task is a term that refers to how a particular task is carried out.

HRI is a wide research area which has been applied in several areas such as entertainment, military, search and rescue missions, education, communication and healthcare.

\section{HUMAN-RoBot INTERACTION IN HEALTHCARE}

The use of robots is swiftly shifting from industrial uses where they are basically deployed for manufacturing purposes and tasks that are too dangerous for human beings to the use of social robots which have the capability to interact with human beings in a particular environment. Social robots have been widely deployed in healthcare in recent times as a result of low accessibility to healthcare services. For instance, in the United States of America, 28.6 million Americans (about 9.1\% of her population) were uninsured in 2015 [31]. One of the major factors responsible for this is cost. Hence, these set of people were hindered from receiving healthcare. This may however lead to loss of lives. Nonetheless, social robots are now being designed to provide affordable home based, personalized and telemedicine technologies for preventive and curative care. In addition, the upsurge in population as well as the shortage in the number of qualified health workers has also necessitated the need for social robots in healthcare. Hence, researches in HRI in healthcare has resulted in the design of social robots that served as companions to patients, provide support for the aged and the sick and serve as assistive aids or mobility assistance to the visually impaired or people with mobility challenges [32]. Studies in HRI in healthcare have also brought about the design of social robots that provide therapy for autistic children in order to improve their social interactions [33]. Consequently, HRI has the capability to improve the quality and accessibility to healthcare services which in turn increases patients' health outcome.

HRI in healthcare is primarily concerned with helping patients improve or monitor their health. Social robots in healthcare have been classified as surgical robots, rehabilitation robots, behavioral therapy robots, companion robots, assistive and supportive robots, physician surrogate, telepresence robots, biorobots, and vital signs monitoring robot.

\section{A. Surgical Assistance Robots}

Surgical assistance robots are robots that allow physicians to perform surgical operations with greater precision. Surgical robots support both face-to-face and remote surgical operations. In face-to-face surgical operations, the physicians and patients are physically present while the human surgeon is not physically present with the patients in remote surgical operations. The use of surgical assistance robots results in minimally invasive surgeries. Surgical assistive robots have been used in urology for prostate cancer [17]. Advantages of surgical robots according to Kefee [17] include increased precision of surgical manipulation, improved vision due to magnification, a more controlled, comfortable and safer environment as well as better ergonometric for the operator. A typical example of a surgical robot is the Da Vinci surgical system. The Da Vinci surgical system is a teleoperated and telepresence system which consists of an end-effector with surgical instruments. Da Vinci surgical system was designed to emulate a human like wrist with greater flexibility in order to assist surgeons to perform delicate and complicated operations. It is however worthy to note that the Da Vinci surgical system has performed more than 20,000 surgeries [15]. Fig. 3 shows the picture of Da Vinci system. Another type of a surgical robot is the Magnetic Microbots which have been used for removing plaques from a patient's arteries.

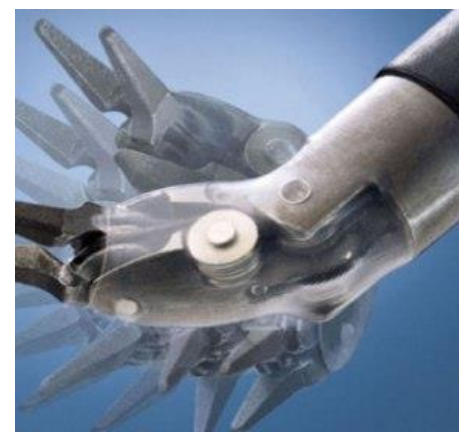

Fig.3. Da Vinci system [17]

\section{B. Rehabilitation Robots}

Rehabilitation robots according to Van der Loos and Reinkensmeyer [2] are robots that assist people with disability and provide therapy for people seeking to improve physical or cognitive functions. According to Van der Loos and Reinkensmeyer [2], disability means a physical or mental impairment that substantially limits one or more of major life activities. Van der Loos and Reinkensmeyer [2] categorized rehabilitation robots as assistive robots for mobility, assistive robots for manipulation and therapy robots.

- Assistive robots for mobility: These are robots that assist people with motor impairments such as difficulty in vision and walking. Examples of assistive robots for mobility include robotic wheelchairs, intelligent wheel chairs, robotic walkers and robotic aids for the blind. Assistive robots improve mobility and navigation and also prevent collision and falls. A typical example of an assistive robot used in healthcare is Pearl. Pearl is a mobile robot system developed as a part of the Nursebot project at Carnegie Mellon University. It is designed to assist elderly people in navigating their daily activities in their environment and also to remind them to take their medications [33]. Pearl is equipped with sonar sensors, microphones 
for speech recognition, speakers for speech synthesis, touch-sensitive graphical displays, actuated head units, and stereo camera systems [33]. Pearl supports telepresence which allows physicians to interact with remote patients. Another example of an assistive robot in healthcare is Robear. Robear is a giant gentle bear with a cartoonish head. Robear has the capability of lifting and transferring patients with mobility problems. Robear can also help patients to stand and turn in bed so as to prevent bed sores. Fig. 4 shows Robear assisting medical practitioners to lift a patient unto a bed.

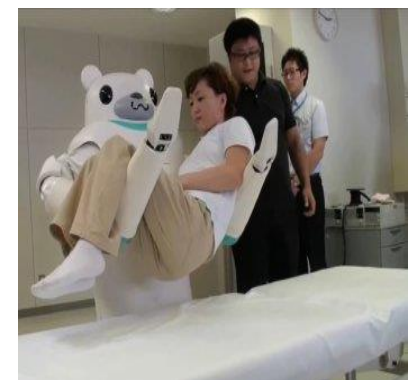

Fig.4. Robear lifting a patient unto a bed [17]

- Assistive robots for manipulation: Assistive robots for manipulation are used for handling physical objects. They are usually used by people with impairments of the arm, hand and fingers. Examples of assistive robots for manipulation include the MANUS robot arm, ISAC robot and Bestic Arm. Bestic arm is designed for lifting food from the plate to the mouth [34]. Handy 1 robot is also a manipulation robot which is used for assisting disabled people with eating and drinking [35]. Fig. 5 shows the picture of Bestic arm.

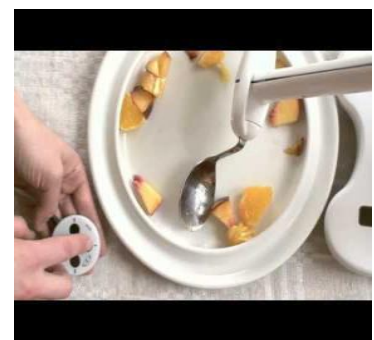

Fig.5. Bestic arm lifting food from a plate [17]

- Therapy Robots: Therapy robots are robots that provide treatment for people with physical and mental challenges. For instance, researches have shown that people suffering from Autism Spectrum Disorders (ASD) responded to treatments involving robotic technology than treatments from human therapists [36]. A typical example of a therapy robot is Paro. Paro was developed by the Intelligent Systems Research Institute (ISRI) of the National Institute of Advanced Industrial Science and Technology (AIST) in Japan. Paro resembles a baby harp seal.
Paro is covered with soft artificial fur and it has been used as a substitute for animal therapy [37]. Paro is not mobile; but it has been used for the treatment of patients with dementia [3]. According to Riek [37], therapy using Paro include patients' holding, hugging, stroking, or talking to Paro as they would an actual animal or baby. Thus, Paro improved patients' social interaction and moods which improves patient's health outcome. Autom, the weight loss coach is also a therapy robot that helps people to reduce their weight by encouraging diet adherence and exercise [38]. Pepper, a four feet humanoid robot is also a therapy robot developed by Softbank to improve the mental engagement of humans by responding to their emotions.

\section{Companion Robots}

Companion robots are typically designed to enhance the health and psychological well-being of the aged and the sick by providing companionship, alleviating stress and increasing their immune system. Companion robots have been used to increase the quality of life and the life span of the aged who usually lack human care and support in the society. Hence, companion robots mitigate helplessness, boredom, isolation, depression and loneliness [3]. Paro has also been used as a companion robot. In addition, AIBO, a metallic doglike robot has been used as a companion robot in nursing homes. A study conducted by Banks et al. [39] showed that AIBO reduces stress hormones and also improve the brain functioning of patients.

\section{Entertainment Robots}

These are robots that improve the health and wellbeing of patients by entertaining them. Examples of entertainment provided by these types of robots include games, music and video. A typical example of an entertainment robot used in healthcare is the Guide robot manufactured by ED Robotics Company in Seoul, Korea [3]. The Guide robot also takes the vital signs of its users. Guide robot interacts with its users by speaking, displaying messages, images, videos and texts on a touch screen. It also accepts users' input on the touch screen [3]

\section{E. Telepresent Robots}

A telepresent robot is a form of telemedical robot that allows healthcare professionals that are offsite or in remote locations to participate in the care of a patient. Telepresent robots are however non-autonomous in nature and they are basically designed to facilitate remote communication as well as the timely treatment of patients. Telepresent robots are typically used for guiding therapy from remote locations. A typical example of a telepresent robot is Dr. Robot. Dr. Robot according to Kefee [17] allows remote neurologists to provide special care for patients with acute stroke in an emergency room. Another example of a telepresent robot is the RP-VITA robot developed by In touch health and iRobot. RP-VITA robot is designed to collect patients' data and disseminates it to 
designated healthcare professionals. However, whenever there is an abnormality in the data collected, RP-VITA robot notifies the healthcare professionals through alerts [17].

Table 1 shows a comparative analysis of social robots in healthcare.

Table 1. A Comparative Analysis of Some Social Robots in Healthcare

\begin{tabular}{|c|c|c|c|}
\hline $\begin{array}{c}\text { Healthcare } \\
\text { Robot }\end{array}$ & $\begin{array}{c}\text { Types of } \\
\text { Interaction }\end{array}$ & $\begin{array}{c}\text { Level of } \\
\text { Autonomy }\end{array}$ & $\begin{array}{l}\text { Interaction } \\
\text { Roles }\end{array}$ \\
\hline $\begin{array}{l}\text { Da Vinci } \\
\text { Surgical } \\
\text { System }\end{array}$ & $\begin{array}{c}\text { Proximate } \\
\text { and Remote }\end{array}$ & $\begin{array}{c}\text { Non- } \\
\text { Autonomous }\end{array}$ & $\begin{array}{c}\text { Human is } \\
\text { Supervisor } \\
\text { and Operator } \\
\text { Humans and } \\
\text { Robot are } \\
\text { Team }\end{array}$ \\
\hline $\begin{array}{c}\text { Robotic } \\
\text { Wheelchair }\end{array}$ & Proximate & $\begin{array}{c}\text { Non- } \\
\text { Autonomous }\end{array}$ & $\begin{array}{c}\text { Humans and } \\
\text { Robots are } \\
\text { Team }\end{array}$ \\
\hline Pearl & $\begin{array}{l}\text { Proximate } \\
\text { and Remote }\end{array}$ & $\begin{array}{c}\text { Non- } \\
\text { Autonomous }\end{array}$ & $\begin{array}{c}\text { Human is } \\
\text { Supervisor } \\
\text { Humans and } \\
\text { Robot are } \\
\text { Team }\end{array}$ \\
\hline Paro & Proximate & $\begin{array}{c}\text { Non- } \\
\text { Autonomous }\end{array}$ & $\begin{array}{c}\text { Humans and } \\
\text { Robots are } \\
\text { Team } \\
\end{array}$ \\
\hline Guide & Proximate & $\begin{array}{c}\text { Non- } \\
\text { Autonomous }\end{array}$ & $\begin{array}{c}\text { Humans and } \\
\text { Robots are } \\
\text { Team }\end{array}$ \\
\hline Autom & Proximate & $\begin{array}{c}\text { Non- } \\
\text { Autonomous }\end{array}$ & $\begin{array}{c}\text { Humans and } \\
\text { Robots are } \\
\text { Team }\end{array}$ \\
\hline
\end{tabular}

\section{Challenges of Human-Robot INTERACTION IN HEALTHCARE}

Social robots are now widely used in healthcare. Their applications range from surgery, emotional and aging care, companionship to telemedicine and rehabilitation. However, there are numerous challenges associated with the interaction between humans and social robots in healthcare. These challenges range from ethical challenges, design issues to safety, usefulness, acceptability and appropriateness.

\section{A. Ethical Challenges}

Ethics is a philosophical discipline which is concerned with the morality of human behavior, with right and wrong [40]. There are four basic principles of healthcare ethics. These principles were developed by Tom Beauchamp and James Childress [41]. These four principles include autonomy, beneficence, nonmalfeasance and justice. The ethical challenges confronting HRI in healthcare are discussed in line with these four principles.

- Autonomy: Autonomy in this regard refers to the right of a patient to have control over his or her body. Hence, patients are allowed to make decisions concerning their health. Autonomy also refers to the right of a patient to have the knowledge of their health information and a right to their healthcare [42]. Thus, patients must be fully aware before their health information is exchanged amongst diverse healthcare providers [43]. For instance, some robots are designed to assist in the monitoring of patients health during human-robot interaction. Such robots may have cameras installed on them which enable healthcare providers to monitor their patients' health remotely. Some of these robots may have the ability to record and transmit data in humanreadable format [7]. This usually causes privacy concerns especially when the patient is not aware of whom the data is transmitted to. This is because patient's information must not be divulged or revealed to anyone who is not involved in the care of the patient. This may lead to lack of confidence and trust in healthcare providers, and this can prevent patients from disclosing relevant information at the point of care. Nevertheless, trust is essential in HRI in healthcare as it affects the willingness of the healthcare providers and patients to accept the information and suggestions generated by the robots which invariably affects the decision making process of the healthcare system.

- Beneficence: Beneficence simply means to do good. The principle of beneficence ensures that all procedures and therapy are done to ensure the well being of patients. To ensure beneficence in healthcare, healthcare professionals must maintain a high level of skills and knowledge in the use of current and best medical practices. However, one of the major challenges facing HRI in healthcare is the high cost involved in training healthcare professionals in the use of robots for therapy and assistive care. Furthermore, the cost of maintaining social robots in healthcare is high. Hence, social robots are not widely deployed in healthcare despite their significant impacts on healthcare delivery.

- Non-Malfeasance: Non-malfeasance or primum non nocere in Latin means to do no harm [44]. Harm in this definition refers to anything which worsens the conditions of patients such as the introduction of pain, discomfort, suffering, disability or disfigurement and death [43]. However, if a robot is not designed with safety in mind, it could harm the users it is designed to interact with [6]. Hence, social robots in healthcare such as robotic wheelchairs and walkers must be designed to avoid obstacles, collision and fall while maneuvering in an environment. In addition, during HRI, humans and robots exchange information and energy. Nevertheless, the transmission of too much energy by the robot to the human may result in severe injury. Furthermore, the presence of sharp edges in the mechanical design of a social robot can result in lacerations which can also cause severe damages to humans [9]. In addition, in HRI, anthropomorphism creates emotional attachment 
between humans and robots. This however can create a degree of deception in the minds of humans. This is because when patients are emotionally attached to anthropomorphic robots, they might begin to think they are truly humans. Thus, a loss or an irreparable damage of the robot that a patient is emotionally attached to can result in the deterioration of the patient's health. Furthermore, the emotional attachment between humans and robots is unidirectional [45]. Hence, social robots cannot reciprocate the emotions accorded them by humans. In addition, some humans may exhibit decreased trust and negative emotional responses towards some robots that imperfectly resemble human beings. Sparrow and Sparrow [46] argued that the use of robots to provide care to the vulnerable especially older people would most likely result in the reduction of human contact which is detrimental to their wellbeing. Another challenge confronting humanrobot interaction is the ability of the robot to fail or malfunction during healthcare delivery process. This is however harmful to the health of the patients.

- Justice: The principle of justice ensures that there is fairness in decisions concerning the care of a patient. It also ensures the fair distribution of scarce medical resources amongst patients. The principle of justice also ensures that appropriate laws and legislations that ensure data availability, privacy, confidentiality, accuracy, integrity, accountability and security are put in place during healthcare delivery. In HRI, security and privacy issues such as unauthorized view of patients' information is still a major cause of concern.

\section{B. Usability Challenges}

The International Standard Organization (ISO) 924111 defines usability as the extent to which a product can be used by specified users to achieve specified goals with effectiveness, efficiency and satisfaction in a specified context of use [47]. Hence, usability can be described as how easy it is for users to accurately and efficiently accomplish a task while using a system. Usability is concerned with a system's particular users, their tasks and the system's environment of use. Mayhew [48] defines usability based on how well a system supports the user's real life tasks, how easy it is for diverse user groups to learn the use of a system, how efficient the system is for frequent users, how easy it is for occasional users to remember the functionalities of the system, how satisfied the users are with the system and how easy it is for the system users to understand what the system does. The following are some of the usability challenges confronting HRI in healthcare.

- User/Social Acceptance: Dillion [49] defines user acceptance as the demonstrable willingness within a user group to employ a technology for the tasks it is designed to support. In HRI, social acceptance is defined as an individual's willingness to integrate a robot into an everyday social environment based on interaction and experiences [50]. For instance, one of the challenges facing the Sedasys system, a social robot that delivers anesthesia to patients without an anesthesiologist, is low social acceptance rate. This is due to the fear of autonomous care that the robot provides [17]. Hence, the fear of displacement of healthcare professionals by care robots is heightened.

- User Experience: User experience in humanrobot interaction according to Weiss [50] deals with the way people use the interactive product, the way it feels like in their hands, how well they understand how it works, how they feel about it while they are using it, how well it serves their purposes, and how well it fits into the entire context in which they are using it. Nonetheless, Riek [37] reported that most upper-limb rehabilitative robots are so difficult for therapists to use and as a result they remain dormant in closets after they are purchased. In addition, the study carried out by Hayley [3] showed that the sound made by Paro was distressing to some of its users and hence they disliked it.

- Culture: Culture in this parlance refers to the ethnic, national or geographic location of the users of the robots. It also encompasses the religion, language and cultural values of the users of the robots. For instance, the way the Japanese or South Koreans interact with robots is quite different from the way the Europeans interact with them. The Japanese are usually more enthusiastic in the deployments of robots than the Europeans. This is because the use of automatons has a long tradition in religious ceremonies in Japan, and also the positive presentation of robots in Japanese literature leads to a high acceptance of robots in Japan [50]. Conversely, the Middle Eastern culture is opposed to iconic technologies, such as humanoid robots [37]. Hence, the use of social robots in the Middle East is limited.

- Attitude towards the use of the Technology: Venkatesh et al. [51] defines attitude as an individual's overall affective reaction to using a system. May et al. [52] conducted a study on the attitude of healthcare providers on the use of a robotic telepresent psychiatric treatment delivery system. The result of the study showed that some of the healthcare providers felt that the presence of the robotic technology during care was a threat to the healthcare delivery process. Conversely, a study carried out by Hayley et al. [3] showed that users were enthusiastic and had positive attitude towards the use of Paro because the users believed that Paro was beautiful looking, life-like, tactile and had lovely eyes.

- Robot Morphology: This refers to the form or the appearance of the robot. Social robots can be 
mechanical or anthropomorphic in appearance. However, when a robot's appearance is very close to that of a human, the feelings of comfort and familiarity declines. This concept is referred to as the uncanny valley. For instance, Pino et al. [53] emphasized that hyper-realistic representations of robots with human appearance could lead persons with dementia to confusion. Hence, the use of such robots in the provision of therapy for patients is a challenge. In addition, Riek et al. [37] conducted a study on the attitudes of humans towards humanoid robots in the United Arab Emirates where the use of iconic technology is discouraged. Riek et al. [54] used a humanoid robot that resembles the philosopher Ibn Sina in their study. The result of the study showed that despite the opposition to the use of iconic technology in United Arab Emirates, the humanoid robot was well-accepted when used for healthcare services because of its appearance.

\section{RECOMMENDATIONS FOR ENHANCING HUMAN-ROBOT INTERACTION IN HEALTHCARE}

This research so far has shown that social robots have broad applications in healthcare. They are used to provide therapeutic and assistive care to the vulnerable, they are also used to provide companion to patients and they also assist in surgical operations. The interaction between humans and social robots in the healthcare system is no doubt bedeviled with numerous challenges ranging from emotions and deception, safety, privacy to lack of trust, high cost of training healthcare professionals in the use of robotic technology as well as usability issues. The major consequence of these challenges is a decline in the acceptance rate of the robotic technology in healthcare. However, the following suggestions can be adopted to enhance an effective interaction between humans and robots in healthcare.

- The care of a patient should not be totally left in the hands of an autonomous social robot. Social robots should be made to provide complementary care and not replace human contacts. This will prevent patients from being totally emotionally attached to social robots.

- Healthcare laws and security policies such as the Health Insurance Portability and Accountability Act (HIPAA), National Health Information Technology and Privacy Advancement Act of 2007, Technologies for Restoring Users Security and Trust in Health Information Act of 2008, Red Flags Rule as well as The American Recovery and Reinvestment Act (ARRA) should be implemented in HRI in healthcare. This will prevent breaches of sensitive healthcare information and also ensure that patients have legal rights concerning their personally identifiable healthcare information. This will also ensure that patients have a right to how their information are revealed and used for other purposes apart from treatment, payment and other medical operations. Hence, policies that ensure patients' consent should be encouraged when robots are involved in the exchange of patients' data.

- Human factors should be considered during the design of social robots because humans are considered as a major component in human-robot interaction. For instance, when designing robots to aid the movement of older people, the robot designer should consider that most elderly people are slow in movement and are weak. Hence, robots with slower motion and soft surface should be considered for elderly people.

- The safety of both the robot, patient and healthcare providers should be considered during humanrobot interaction. Robots with sharp edges that can cause lacerations to patients should be avoided in healthcare. Safety devices such as safety screens should also be employed in the design of assistive mobile robots in order to prevent collision and fall.

- The uncanny valley should be avoided during the design of humanoid robots in healthcare. Hence, the appearance of humanoid robots in healthcare should not be too close to that of human beings in order to avoid a repulsive reaction from patients and caregivers. This will prevent the expression of fear by its users.

- Trust is very essential in human-robot interaction. This is because the lack of trust in the interaction between human beings and robots could result in the misuse and abuse of robots. Hence, trust facilitates the reliance of human beings on the ability of social robots to perform their tasks. Hence, trustworthy robots should be designed for the healthcare system. This will help social robots used in healthcare to perform their tasks effectively.

- Social robots in healthcare should be designed to be emotionally intelligent. They should be able to recognize and understand human emotions as well as respond to and manage these emotions. This is because emotionally intelligent robots are less frustrating to deal with [55]. For instance, Cameron [56] is of the view that a robot that is able to recognize a human emotion can modify its own behavior to be more accommodating.

- The integration of social robots into the healthcare system is a challenging task. This is because users perceive autonomous robots differently from other computer technologies. Social robots conform to the rules of their environment and also negotiate their interactions with human beings [50]. Nevertheless, social robots within the context of healthcare must be robust, effective, efficient and flexible. This will enhance a human-robot team in the healthcare system to accomplish a task.

- Social robots in healthcare should be fault tolerant and they should also be designed to degrade gracefully. This is because the frequent failure or 
malfunctioning of social robots during healthcare delivery process can be harmful to the health of patients.

Hence, it is advised that social and ethical implications of social robots should be considered during the design of social robots in healthcare.

\section{CONCLUSION}

Human-robot interaction is an emerging and dynamic field which deals with the interaction between humans and social robots in a specific environment. Human-robot interaction has been applied in several fields such as Education, Military, Entertainment, Communication and Healthcare. Human-robot interaction is fast becoming popular in healthcare as a result of the increase in the number of vulnerable populations, rising cost of healthcare and the shortage of qualified healthcare professionals. HRI have been used to provide companionship, surgical operations, rehabilitative care and entertainment to humans within the context of healthcare. Consequently, HRI improves patient health outcome as well as the quality of healthcare services delivered to patients. In spite of the numerous benefits of social robots in healthcare, the interactions between humans and robots are bedeviled by numerous challenges. These include privacy, safety, the form of the robot, trust, emotions and deception as well as culture. The basic consequence of these challenges is a decline in the acceptance rate of social robotic technology in healthcare. It is against this backdrop that this paper examines the general concepts of robots and their classification based on the task they perform. This study also appraises the concept of human-robot interaction within the concept of healthcare. Ethical and usability challenges affecting human-robot interaction in healthcare are also examined in this paper. The ethical challenges were viewed in line with the principles of ethics designed by Beauchamp and Childress. This study suggests that human factor, privacy, patients' consents as well as the safety of the robots, healthcare providers and patients should be considered during human-robot interaction in healthcare.

\section{REFERENCES}

[1] K. Wada, and T. Shibata, "Living with seal robots in a care house devaluations of social and physiological influences," IEEE/RSJ International Conference on Intelligent Robots and Systems, Beijing, October 2006.

[2] P. S. Lum, C. G. Burgar, P. C. Shor, M. Majmundar, and M. Van der Loos, "Robot-assisted movement training compared with conventional therapy techniques for the rehabilitation of upper-limb motor function after stroke," Archives of Physical Medicine and Rehabilitation, vol. 83, pp. 952-959, 2002.

[3] H.Robinson, A.M. Bruce, N. Kerse, E. Broadbent, "Suitability of healthcare robots for a dementia unit and suggested improvements," JAMDA, Elsevier, pp.1-7. 2012

[4] M.M.A. de Graaf, S. B. Allouch, and J.A.G.M. van Dijk, "What makes robots social?: A user's perspective on characteristics for social human-robot interaction",
Springer-Verlag Berlin Heidelberg, 2015

[5] A.Weiss, and V. Evers, "Exploring cultural factors in Human-Robot Interaction: A matter of personality?," Comparative Informatics Workshop, December, 2011.

[6] D. Feil-seifer, and M.J. Mataric, "Ethical principles for socially assistive robotics". Robotics and Automation Magazine, vol. 18, 2011.

[7] K. S. Bankston, and A. Stepanovich,'When robot eyes are watching you: the law \& policy of automated communications surveillance", We Robot, University of Miami, 2014.

[8] M.Matarić, A.Tapus, C. Winstein,and J. Eriksson, "Socially assistive robotics for stroke and mild TBI rehabilitation", Advanced Technologies in Rehabilitation, vol. 145,pp.249-262, 2008.

[9] A. De Santis, B. Siciliano, A. De Luca, and A. Bicchi, "An atlas of physical human-robot interaction", Mechanism and Machine Theory, Science Direct, Elsevier, 2007.

[10] K. Capek, "Rossum's universal robots,"Dover Publications", 2001.

[11] V. Kumar,'Introduction to Robotics," University of Pennsylvania, Philadelphia", 2014.

[12] Y. S. Choi, "A Study of Human-Robot Interaction with an assistive robot to help people with severe motor impairments", Unpublished PhD Thesis, H. Milton Stewart School Of Industrial And Systems Engineering, Georgia Institute Of Technology, 2009.

[13] A. Davison, "Introduction to robotics," Department of Computing, Imperial College London, 2016.

[14] F. Hegel, C. Muhl, B. Wrede, M. Hielscher-Fastabend, G. Sagerer, "Understanding social robots, "Advances in Human Computer Interaction, 2009.

[15] M. O. Qureshi, and R.S. Syed, "The impact of robotics on employment and motivation of employees in the service sector, with special reference to health care," Safety and Health at Work, vol. 5, pp. 198-202, 2014.

[16] S.Kiesler, and P. Hinds,"Introduction to this special issue on Human-Robot Interaction,"Special Issue of HumanComputer Interaction, vol. 19, 2004.

[17] B. Keefe, "Robots/robotics in healthcare", Mayo Clinic Center for Innovation, 2015.

[18] A. Bruce, I. Nourbakhsh, R. Simmons, "The role of expressiveness and attention in human robot interaction", Fall Symposium on Emotional and Intelligence. II, The Tangled Knot of Social. Cognition, 2001.

[19] K. G. Engelhardt, R. A. Edwards, "Human robot integration for service robotics," in Human-Robot Interaction, Mansour Rahimi, Waldemar Karwowki. Eds. London: Taylor \& Francis Ltd., 1992, pp. 315-346.

[20] C. Bartneck, J. Forlizzi, "A design-centred framework for social human-robot interaction," Proceedings of RO-MAN, Kurashiki. pp. 591-594, 2004.

[21] C. Breazeal, Designing Sociable Robots, Cambridge: MA, MIT Press, 2002.

[22] T. W. Fong, I. Nourbakhsh, I, K. Dautenhahn, "A survey of socially interactive robots: Concepts, design, and applications," Robotics and Autonomous Systems, vol. 42, pp. 142-166, 2002.

[23] J. Złotowski, D. Proudfoot, K. Yogeeswaran and C. Bartneck, "Anthropomorphism: opportunities and challenges in human-robot interaction," International Journal of Social Robots, vol. 7, pp. 347-360, 2015.

[24] I. Asimov, "I, Robot," Doubleday, 1950.

[25] J. Vincent, S. Taipale, B. Sapio, G. Lugano, and L.Fortunati, "Social robots from a human perspective," Springer, 2015. 
[26] M. A. Goodrich and A. C. Schultz, "Human-robot interaction: A survey," Foundations and Trends in Human-Computer Interaction, vol. 1, pp. 203-275, 2007.

[27] A. Weiss, R. Bernhaupt, M. Tscheligi, and E. Yoshida, "Addressing user experience and societal impact in a user study with a humanoid robot, Proceedings of the Symposium on New Frontiers in Human-Robot Interaction, 2009.

[28] H.A Yanco, and J. Drury, "Classifying human-robot interaction: an updated taxonomy," Systems, Man and Cybernetics, vol.3, pp.2841-2846, 2004.

[29] J.L. Drury, J. Scholtz, and H.A. Yanco, "Awareness in human-robot interactions," Proceedings of the IEEE Conference on Systems, Man and Cybernetics, vol. 1, pp. 111-119, 2003.

[30] G. Randelli, "Improving human-robot awareness through semantic-driven tangible interaction," Unpublished PhD Thesis, Sapienza University of Rome, 2011.

[31] R.A.Cohen, Health insurance coverage: Early release of estimates from the national health interview survey, National Health Interview Survey Program, http://www.cdc.gov/nchs/data/nhis/earlyrelease/insur2016 05.pdf, 2015.

[32] M. Montemerlo, J. Pineau, N. Roy, S. Thrun, and V. Verma, "Experiences with a mobile robotic guide for the elderly," National Conference on Artificial Intelligence," 2002.

[33] M. E. Pollack, L. Brown, D. Colbry, C. Orosz, B. Peintner, S. Ramkrishnan, S. Engberg, J. Matthews, J. Dunbar Jacob, C. McCarthy, S. Thrun, M. Montemerlo, J. Pineau, and N. Roy," Pearl: A mobile robotic assistant for the elderly," AAAI Workshop on Automation as Caregiver, 2002.

[34] Bestic. "Bestic," http://www.bestic.se/en/home/, 2013.

[35] M. Topping, and J. Smith, "The development of Handy 1, a rehabilitation robotic system to assist the severely disabled," Industrial Robot, vol. 25, pp. 316-320, 1998.

[36] J. J. Diehl, C. R. Crowell, M. Villano, K. Wier, K. Tang, and L. D Riek, "Clinical applications of robots in autism spectrum disorder diagnosis and treatment," Comprehensive Guide to Autism, Springer, pp. 411-422, 2014.

[37] L. D Riek, Robotics technology in mental health care,"in Artificial Intelligence in Behavioral Health and Mental Health Care, D. Luxton (Ed.), Elsevier, 2015.

[38] C. D. Kidd, and C. Breazeal, "Robots at home: Understanding long-term human-robot interaction. IEEE/RSJ International Conference on Intelligent Robots and Systems (IROS), 2008.

[39] M. R. Banks, L. M. Willoughby, and W. A. Banks, "Animal-assisted therapy and loneliness in nursing homes: Use of robotic versus living dogs, "Journal of the American Medical Directors Association, vol. 9, pp. 173177, 2008.

[40] F. Itulua-Abumere, "Ethical issues in health and social care profession," OSR Journal of Humanities and Social Science (JHSS), pp. 14-18, 2012

[41] T.L. Beauchamp, and J.F. Childress, Principles of Biomedical Ethics, New York: Oxford University Press, 1994.

[42] P.S Winkelstein, "Ethical and social challenges of electronic health information,"Medical Informatics, pp.139- 159, 2009.

[43] O.G. Iroju and J.O. Olaleke," Ethical issues in interoperability of electronic healthcare systems," Communications on Applied Electronics, New York, USA, pp. 12-18, 2015.
[44] M. Almacen, "EHR interoperability: legal, ethical, and social challenges," Northwestern University Evanston, Illinois, 2013.

[45] M. Scheutz,"The inherent dangers of unidirectional emotional bonds between humans and social robots," Human-Robot Interaction Laboratory Cognitive Science Program, Indiana University Bloomington, USA, 2015

[46] R. Sparrow, and Sparrow, L., "In the hands of machines? The future of aged care," Minds Machines, vol. 16, pp. 141-161, 2006.

[47] ISO 9241-11," Ergonomic requirements for office work with visual display terminals - part 11," Guidance on usability, International Organization for Standardization, 1998

[48] D. J. Mayhew, "The usability engineering lifecycle: A practitioner's handbook for user interface design," Morgan Kaufman Publishers, San Francisco, California, 1999.

[49] A. Dillon, 'User acceptance of information technology," in Encyclopedia of Human Factors and Ergonomics, vol. 1. W. Karwowski, Taylor and Francis, Eds London, 2001.

[50] A.Weiss, R. Bernhaupt, M. Lankes and M. Tscheligi, "The USUS evaluation framework for human-robot interaction," HCI and Usability Unit, ICT\&S Center, University of Salzburg, Sigmund, Haffner-Gasse ,Salzburg, Austria, 2015.

[51] V. Venkatesh and F. D. Davis, "A theoretical extension of the technology acceptance model: Four longitudinal field studies," Management Science, vol. 46, pp. 86-204, 2000.

[52] C. May, L. Gask, T. Atkinson, N. Ellis, F. Mair, and A. Esmail, "Resisting and promoting new technologies in clinical practice: The case of telepsychiatry", Social Science and Medicine, vol. 52, pp.1889 1901, 2001.

[53] M. Pino, M. Boulay, F. Jouen and A.S. Rigaud, "Are we ready for robots that care for us?" Attitudes and opinions of older adults toward socially assistive robots, " Frontiers in Aging Neuroscience, vol.7, 2015.

[54] L. D. Riek, N. Mavridis, S. Antali, N. Darmaki, Z. Ahmed and Al-Neyadi, M.. "Ibn Sina steps out: Exploring Arabic attitudes toward humanoid robots," Proceedings of the Second International Symposium on New Frontiers in Human-Robot Interaction, pp. 88-99, 2010.

[55] R. W. Picard. "What does it mean for a computer to have emotions," Emotions in humans and artifacts, pp 87-102, 2003.

[56] H. Cameron, "On the possibility of robots having emotions. Unpublished PhD Thesis, Depatment of Philosophy, Georgia State University, 2014.

\section{Authors' Profiles}

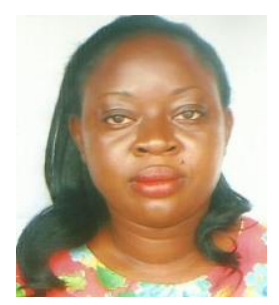

Dr. Iroju Olaronke has a B.Sc. in Computer Technology at Babcock University, Nigeria. She also has M.Sc and $\mathrm{PhD}$ in Computer Science at Obafemi Awolowo University, Nigeria. She bagged a Post Graduate Diploma in Education from Obafemi Awolowo University, Nigeria. She is a member of the Teachers' Registration Council of Nigeria. Dr. Iroju is a lecturer at the Department of Computer Science, Adeyemi College of Education, Ondo, Nigeria. Her research interest is on health informatics, Big Data, humanrobot interaction, interoperability and ontology matching. She has published widely in international journals and conferences 
in the field of Computer Science and she is also a reviewer of several journals. Her professional papers discuss problems in interoperability, usability, ontology, ontology matching, capacity building in electronic healthcare systems and big data in healthcare.

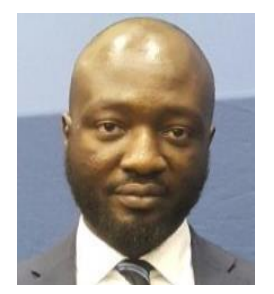

Dr. Oluwaseun A. Ojerinde is a lecturer in the Department of Computer Science in the School of Information and Computer Technology in Federal University of Technology, Minna. He bagged his B.Sc. in Computer Technology at Babcock University in 2006. He received his M.Sc. in Mobile Communication System from Loughborough University in 2008. He also obtained his $\mathrm{PhD}$ in Mobile Communication System from Loughborough University in 2014. His research area is in Antenna, On-body systems, Multiple Input Multiple Output (MIMO) systems, spanning, Telecommunications, Networking and Radiation. He has worked on the effects of metallic objects on radiation for mobile devices. He is a member of IEEE and IET.

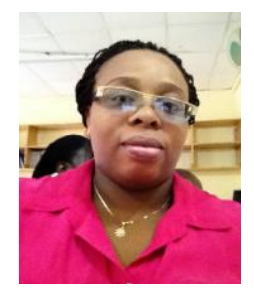

Dr. Ikono R. N. is a Senior Lecturer at the Department of Computer Science and Engineering, Obafemi Awolowo University Ile-Ife. Her research interests are Information System, Health Informatics, Human Robot Interaction, and Software Product Usability. She has published widely in international journals and conferences in the field of Computer Science. She is also a reviewer of several journals. She is a member of IEEE, Nigerian Computer Society, Association for Information Systems Information Development for Health in Africa (INDEHELA) and African Health Informatics in Nigeria.

How to cite this paper: Iroju Olaronke, Ojerinde Oluwaseun, Ikono Rhoda,"State Of The Art: A Study of Human-Robot Interaction in Healthcare", International Journal of Information Engineering and Electronic Business(IJIEEB), Vol.9, No.3, pp.43-55, 2017. DOI: 10.5815/ijieeb.2017.03.06 